\title{
Advanced Backstepping Trajectory Control for Skid-Steered Duct-Cleaning Mobile Platforms
}

\author{
Wootae Jeong ${ }^{1,2 *(\mathbb{D}}$, Seungwoo Jeon ${ }^{3}\left(\mathbb{D}\right.$ and Dahae Jeong ${ }^{2}(\mathbb{D}$ \\ 1 Transportation Environmental Team, Korea Railroad Research Institute, Uiwang 16105, Korea \\ 2 Department of Robotics and Virtual Engineering, University of Science and Technology, Daejeon 34113, \\ Korea; dahaejeong1@gmail.com \\ 3 Defense Robotics Systems Team, Hanwha Defense systems Co., Ltd., Changwon 51575, Korea; \\ sw.jeon@hanwha.com \\ * Correspondence: wjeong@krri.re.kr; Tel.: +82-31-460-5817
}

Received: 28 January 2019; Accepted: 26 March 2019; Published: 4 April 2019

\begin{abstract}
In recent years, a novel skid-steered duct-cleaning mobile platform was developed to remove dust accumulated on the inner surface of an air-ventilation duct with its rolling brushes. During the cleaning process, the irregular brushing pressure acting on the upper arm makes it difficult to control the platform through the duct path. In fact, the repulsive external force due to the brushing pressure is not directly measurable or computable because of the nonlinear deformation of the brush. In addition, dynamic uncertainties in platform motion can occur during reciprocating motion of the upper arm. Therefore, a model-based trajectory-tracking controller is required to control the mobile cleaning platform by considering irregular external forces. The robustness of the developed controller based on the adaptable PD(Proportional-Derivative)-backstepping method has been proposed and evaluated through numerical analysis and experiments. For the turning motion in a narrow space, a skid-steered platform model considering wheel slippage has been also implemented. The result shows that tracking control can be successfully achieved under various conditions of frequencies in brushing-arm motion and torque limitation of the traction motors.
\end{abstract}

Keywords: skid-steering; trajectory tracking; robust control; cleaning robot; mobile platform

\section{Introduction}

Effective air-duct-cleaning techniques have been studied to improve the air quality of ventilation-duct systems broadly used in subway stations and buildings. Automatic control platforms have been developed for duct-cleaning robots and devices to enhance their operability in the dark and limited space inside the duct. Therefore, various ventilation-duct-cleaning robots have been developed with intelligent controllers, sensors, skid-steering functions, and brushing-arm mechanisms. In particular, mechanical brushing methods have been effectively applied for the removal of dusts accumulated inside air-duct surfaces with linkage-arm-based mechanical brushes [1]. The mobile platform also requires a stable trajectory-tracking controller with skid-steering to be used in the limited air-duct space. The rotating brush of the cleaning arm has to consider the interactive forces between the brush and the target surface of the inner duct during the cleaning process. In the dynamic modeling of the mobile platform, the contact forces between the rotating brush and the duct surface induce wheel slippage in the lateral direction, requiring skid-steering control of the platform, as depicted in Figure 1. Therefore, to achieve effective control of the duct-cleaning platform, it is essential to construct a dynamic model of the system including unknown variables such as nonlinear deformation of the filaments of the brush and unknown friction coefficients at each contacting point of the cleaning mechanism. In addition, the lateral loads of the mobile platform and the reciprocating motion of the 
upper-arm brush results in a shifting center of mass $(\mathrm{CM})$ of the platform. The repulsive cleaning forces of the brush and the periodic shift of the CM become dynamic uncertainties of the platform and increase the instability of platform control. Thus, it is essential to develop a robust trajectory-tracking controller that is adaptable for nonlinear systems experiencing complicated interactions during brushing and steering control. Moreover, a trajectory controller that remains stable against uncertainties of the external reaction forces can help to reduce the torque capacity of the traction motors by distributing the torque requirements on each wheel.

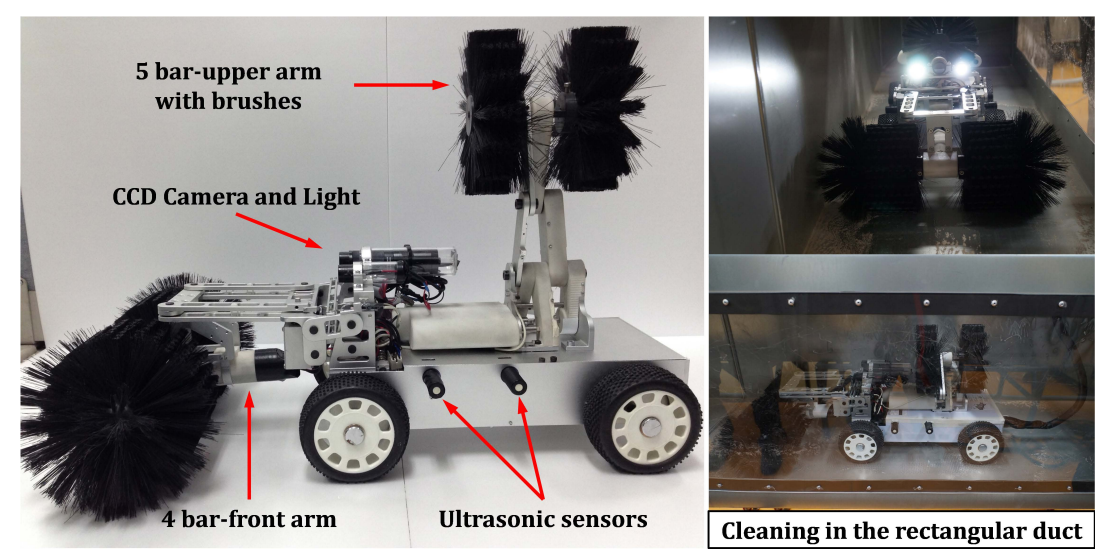

Figure 1. Duct cleaning mobile platform.

Feedback linearization, sliding mode control, and adaptive control methods for nonlinear systems are applicable for the trajectory-tracking control of wheeled mobile platforms constrained by nonholonomic conditions [2-4]. However, linearization techniques are restrictive and often make the system unstable because of chattering issues and high sensitivity to external disturbances [5]. Thus, trajectory planning and tracking control under high dynamic uncertainty requires backstepping controllers that use velocity feedback to stabilize tracking errors asymptotically [6]. In addition, fuzzy inference methods and neural-network algorithms can also provide good solutions for inferring uncertainties whose feedback control is difficult without a complete dynamic model of the controlled system [7,8]. However, intelligent-control schemes can require prior information about the target system or higher computational-processor capability. Therefore, conventional PID controllers have been used to reduce trajectory-tracking errors, rather than nonlinear control [9].

In addition, a conventional backstepping method has been suggested for the nonlinear interacting forced system in the control of duct cleaning mobile platform as a strict feedback form. Therefore, system robustness can be maintained by using high gain control. However, the mobile platform system can be unstable in case of uncertain parameters and un-modeled dynamics. Recent researchers also propose adaptive control [10], sliding mode control [11], and a neural network [12] based on the backstepping control scheme. These approaches can provide solutions for the system with uncertainties and external disturbance, but can increase complexity and processing loads in the real time control system.

The four-wheel skid-steered platform can be modeled for the model based real-time trajectory-tracking. Wheel slippages can also be considered for the skid-steering-motion control of differentially driven platforms. Such slippage can be estimated from the shear stress of the tire-road contact mechanism, but this technique is not preferred for real-time control applications because of very high calculation loads [13]. In addition, the center of curvilinear motion of a skid-steered platform becomes the instantaneous center of rotation (ICR) affected by wheel slippage. In two-wheeled platforms, the ICR can be located on the extended line connecting the left and right wheels. However, the ICR cannot be geometrically described in four-wheeled platforms. Thus, the location of the ICR has been estimated through experiments considering the kinematics of the platform [14,15]. Moreover, the estimation of ICR position is much more difficult in low-speed skid-steered platforms. In this study, an effective modeling and control method for skid-steered duct-cleaning platforms has 
been proposed with various backstepping-based nonlinear controllers. Therefore, we achieve robust trajectory-tracking control for the skid-steered duct-cleaning mobile platform.

\section{Design of the Duct Cleaning Robot}

\subsection{A Mobile Duct-Cleaning Platform}

A simple linkage mechanism with rotating brushes (as depicted in Figure 1) has been applied to the platform to clean the interior duct surface. The brushing arms consist of a four-bar-based front arm and a five-bar-based upper-arm mechanism whose cleaning workspace covers all sides of the duct surface during the cleaning process. In addition, the skid-steering controller implemented by wheel-speed differentials enables effective turning motion within the limited space inside the duct. The front and rear wheels at each side are connected through a timing belt and a pulley module driven by the DC motor. Ultrasonic sensors at each side are used to measure the distance between the mobile platform and the duct wall. A CCD-camera module has been equipped to monitor the cleaning process.

While control strategies using the skid-steering mechanism can be widely used in many applications, they require prediction or estimation of wheel slippage that is changeable depending upon the dynamic environment. Furthermore, the dynamically changing disturbances during interactions between the brush and duct surfaces affect the wheel dynamics and thus tracking control of the mobile platform. Therefore, design of the model-based trajectory-tracking controller for overcoming wheel slippage has become a key feature for the skid-steered duct-cleaning mobile platform.

Figure 1 illustrates the novel duct-cleaning robot and its operating environment in the air duct. A schematic-control diagram of the mobile robot is shown in Figure 2.

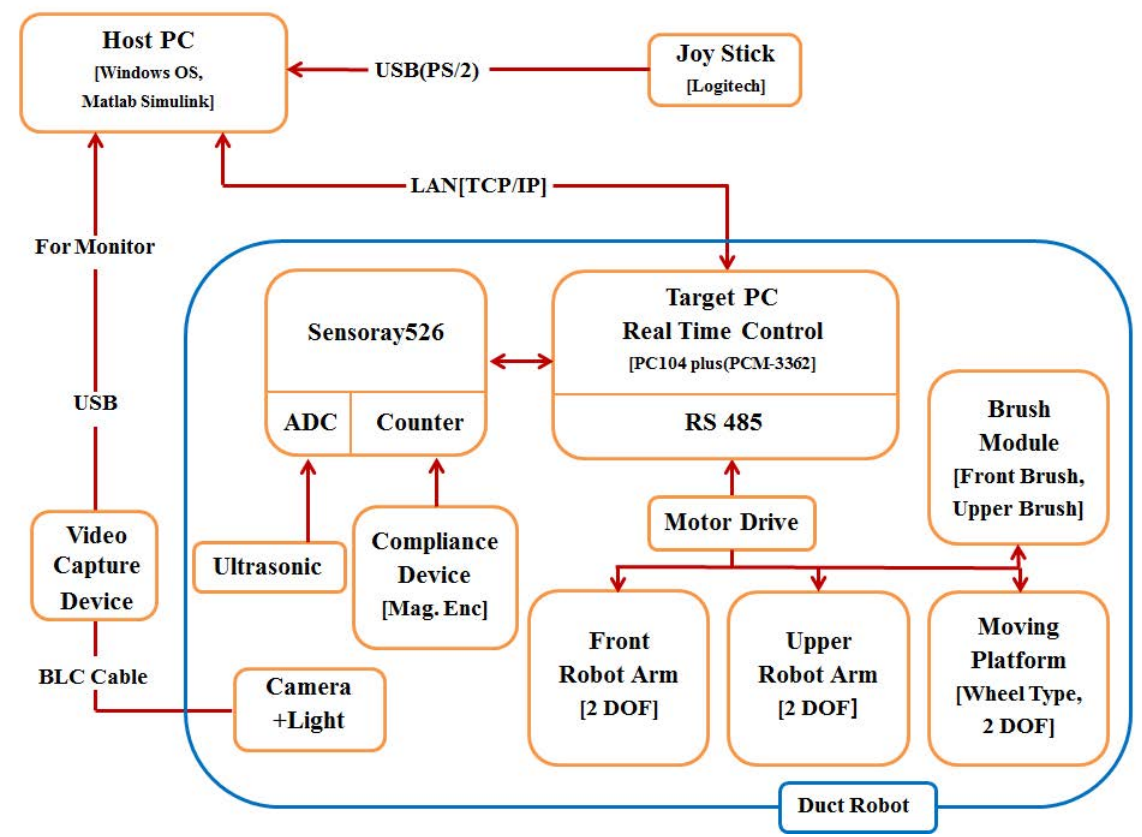

Figure 2. Control system diagram of the duct cleaning platform.

\subsection{Modeling of the Skid-Steered Platform}

The main goal of the four-wheeled mobile platform is to enable accurate trajectory-tracking control while cleaning ducts with its rolling brushes and arm. Therefore, it is essential to estimate the trajectory of the platform for the tracking controller based on a kinematic and dynamic modeling of the platform. The basic model of the wheeled mobile platform is represented by the global coordinate $(q=[X, Y, \Phi])$ as illustrated in Figure 3. Lateral forces acting on each wheel are described as functions 
of the vertical forces at the contacting points with the floor. Based on the model configuration in Figure 3, the equation of motion for the skid-steered platform with coordinate $q=[X, Y, \Phi]$ becomes

$$
\begin{aligned}
& \mathbf{M} \ddot{\mathbf{q}}+\mathbf{F}(\mathbf{q}, \dot{\mathbf{q}})=\mathbf{E}(\mathbf{q}) \tau+\mathbf{A}^{\mathbf{T}}(\mathbf{q}) \lambda \\
& \mathbf{M}=\left[\begin{array}{ccc}
m & 0 & m I C R_{x} \sin \phi \\
0 & m & -m I C R_{x} \cos \phi \\
m I C R_{x} \sin \phi & -m I C R_{x} \cos \phi & I
\end{array}\right] \\
& \mathbf{F}(\mathbf{q}, \dot{\mathbf{q}})=\left[\begin{array}{c}
R_{x} \cos \phi-F_{y} \sin \phi+m I C R_{x} \dot{\phi}^{2} \cos \phi \\
R_{x} \sin \phi+F_{y} \cos \phi+m I C R_{x} \dot{\phi}^{2} \sin \phi \\
M_{r}
\end{array}\right] \\
& \mathbf{E}(\mathbf{q})=\left[\begin{array}{cc}
\cos \phi & \cos \phi \\
\sin \phi & \sin \phi \\
t & -t
\end{array}\right] / r \\
& \tau=2 r F_{x i}, \quad(i=1,2)
\end{aligned}
$$

where $F_{x i}$ is the tractive force at the contact of the wheel, $R_{x}$ is the sum of the longitudinal resistive forces of the wheels, $F_{y}$ is the sum of the lateral forces at the contact patches of the wheels, $\mathrm{r}$ is the wheel radius, $t$ is half of the track width, ICR $R_{x}$ is the longitudinal distance of the ICR at the CM, $\tau_{i}$ are the torques of each driving motor, matrix $\mathrm{A}(\mathrm{q})$ represents the constraint condition, and $\lambda$ is Lagrange multiplier [16]. The quantities $R_{x}, F_{y}$ and $M_{r}$ can be calculated by longitudinal and lateral friction coefficients $\left(\mu_{x}, \mu_{y}\right)$ between the wheel and the floor as follows

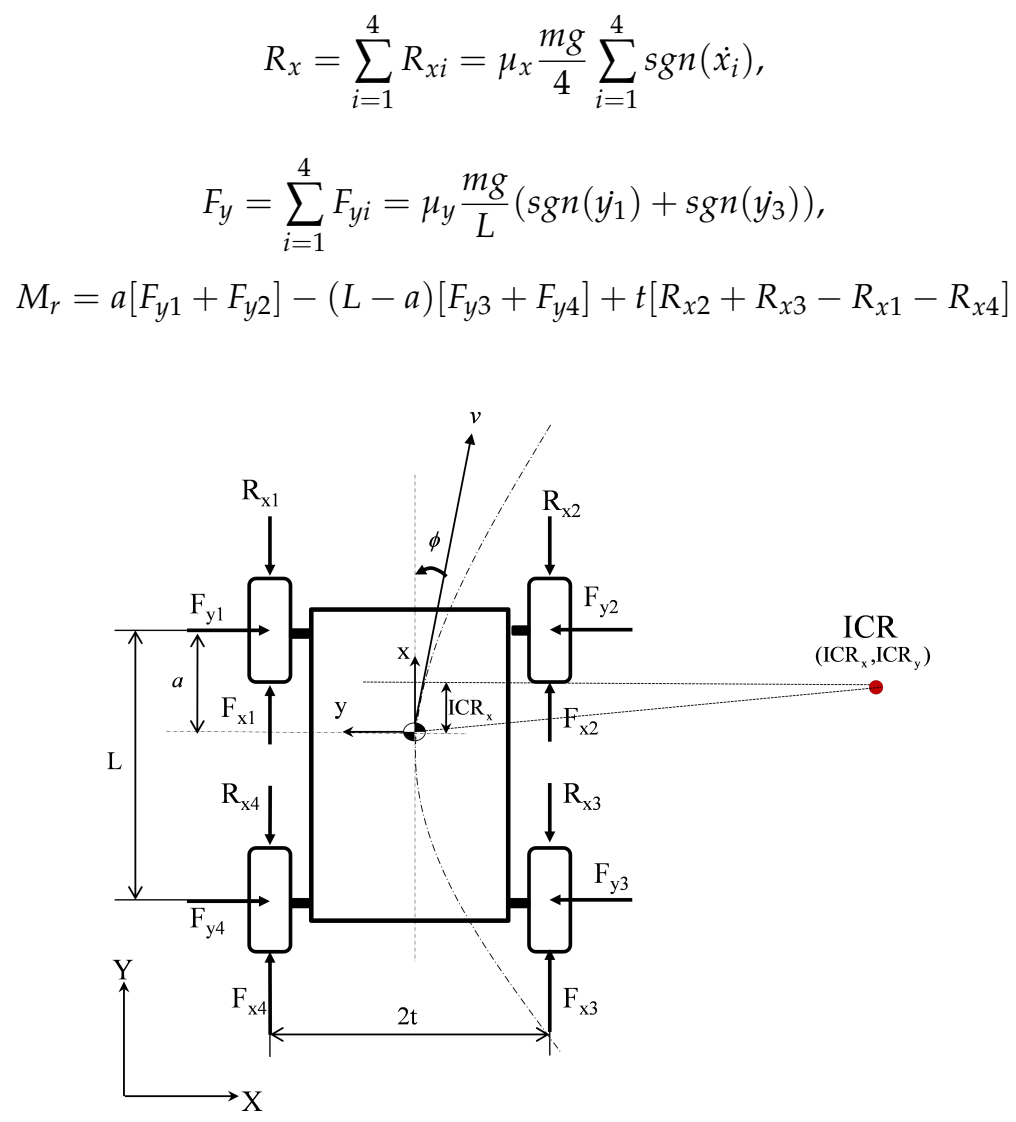

Figure 3. A schematic model of the skid-steered mobile platform. 
The kinematics of the skid-steering platform are described by

$$
\begin{gathered}
\dot{q}=\mathbf{S}(q) \mathbf{v}, \quad \mathbf{v} \in R^{2} \\
\mathbf{S}(\mathbf{q})=\left[\begin{array}{cc}
\cos \phi & -I C R_{x} \sin \phi \\
\sin \phi & I C R_{x} \cos \phi \\
0 & 1
\end{array}\right]
\end{gathered}
$$

where $S(q)$ is $3 \times 2$ coordinate transformation matrix and $\mathbf{v}=\left[\begin{array}{ll}\dot{x} & \omega\end{array}\right]$ is the velocity vector at the $\mathrm{CM}$ of the platform. Nonholonomic constraints can also be applied to the kinematics because the skid-steered platform cannot be driven directly in the lateral direction. The equation of motion considering nonholonomic contraints can be obtained by defining $\mathbf{A}(\mathbf{q})$ [6].

$$
\begin{gathered}
\mathbf{A}(\mathbf{q})(\dot{\mathbf{q}})=\left[\begin{array}{ccc}
-\sin \phi & \cos \phi & I C R_{x}
\end{array}\right]\left[\begin{array}{c}
\dot{X} \\
\dot{Y} \\
\dot{\phi}
\end{array}\right]=0, \\
\mathbf{S}^{T}(\mathbf{q}) \mathbf{A}^{T}(\mathbf{q})=0 \\
\mathbf{M} \ddot{\mathbf{q}}+\mathbf{F}(\mathbf{q}, \dot{\mathbf{q}})=\mathbf{E}(\mathbf{q}) \tau
\end{gathered}
$$

Torques from the controller for trajectory-tracking are calculated from Equation (6) as follows

$$
\tau=\left(S^{T} E\right)^{-1}\left(S^{T} M S u+S^{T} M \dot{S} v+S^{T} F\right)
$$

where $u=\left(u_{1}, u_{2}\right)$ is referred to as the control input.

\subsection{Estimating ICR with a Wheel Slippage Model}

The turning motion of the four-wheel skid-steered platform can be explained at the point of the ICR by the difference in the wheel speed on each side. The ICR can be defined by

$$
I C R_{x}=-\frac{\dot{y}}{\dot{\phi}^{\prime}}, \quad I C R_{y}=\frac{\dot{x}}{\dot{\phi}^{\prime}}
$$

where the longitudinal, lateral, and yaw velocities at the center of mass of the platform are $\dot{x}, \dot{y}$ and $\dot{\phi}$ respectively. In case of two-wheeled platforms, the ICR is located geometrically on the extended line of the virtual driving axle of the two wheels, which passes through the CM of the platform. In the lateral direction, the ICR approaches closer to the platform as the velocity difference between the left and right wheels increases, vice versa, the ICR becomes more distant from the CM as the velocity difference decreases.

However, in case of two driving axled platform, the ICR cannot be located on the line which passes through the $\mathrm{CM}$. The radius of curvature of the platform turning motion is based on the distance from the ICR to the CM, and can be shifted according to the amount of wheel slippage in the differentially driven turning platform. Estimating the position of the ICR under wheel slippage is required to control the trajectory of the skid-steered platform. The longitudinal position of the ICR can be estimated by the sequence using the yaw rate $\left(\dot{\phi}^{\prime}\right)$ of the local coordinate, as shown in Figure 4.

The kinematic matrix, $S^{\prime}(q)$, becomes

$$
\mathbf{S}^{\prime}(\mathbf{q})=\left[\begin{array}{cc}
\frac{1}{2}\left(1-\lambda_{1}\right) \cos \phi^{\prime} & \frac{1}{2}\left(1-\lambda_{2}\right) \cos \phi^{\prime} \\
\frac{1}{2}\left(1-\lambda_{1}\right) \sin \phi^{\prime} & \frac{1}{2}\left(1-\lambda_{2}\right) \sin \phi^{\prime} \\
\frac{\left(1-\lambda_{1}\right)}{2 t} & \frac{\left(1-\lambda_{2}\right)}{2 t}
\end{array}\right]
$$


where the slip ratio $\left(\lambda_{i}\right)$ of wheels is

$$
\lambda_{i}=\left|r \omega_{i}-\dot{x}\right| / r \omega_{i}, \quad(i=1,2,3,4)
$$

with $\omega_{i}$ being the angular speed of the $i^{\text {th }}$ wheel [17].

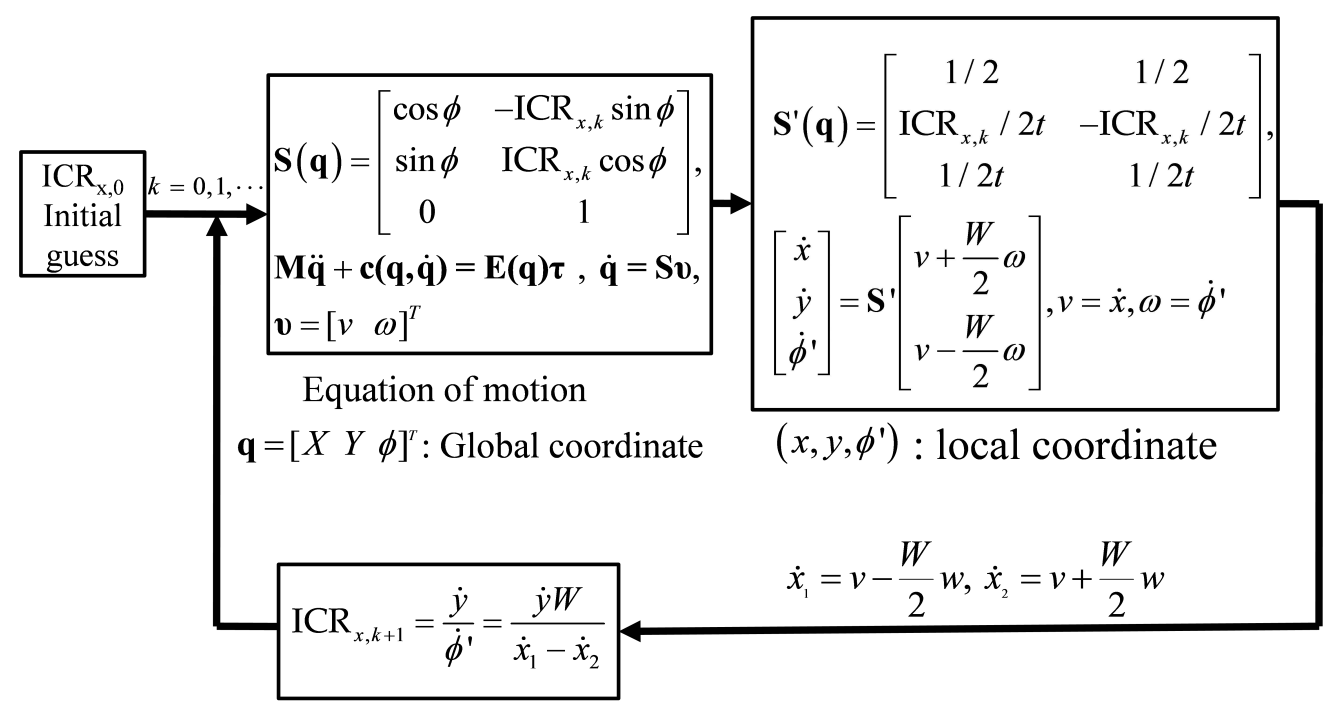

Figure 4. A scheme of the estimating the ICR under wheel slippage.

\subsection{Modeling of the Interactive Forces}

The dust-removal process is carried out by the contact forces between the duct surface and the rotating brush attached at the end of a moving arm on the mobile platform. Since the rotating brush consists of thin filaments whose deformation is irregular during the cleaning process, it is difficult to calculate the friction and repulsive forces of the rotating brush. Nevertheless, those unknown forces have to be estimated under given conditions by modeling the interaction, so as to minimize trajectory errors. Figure 5 shows the free body diagram considering the interacting-force model during the brushing process.

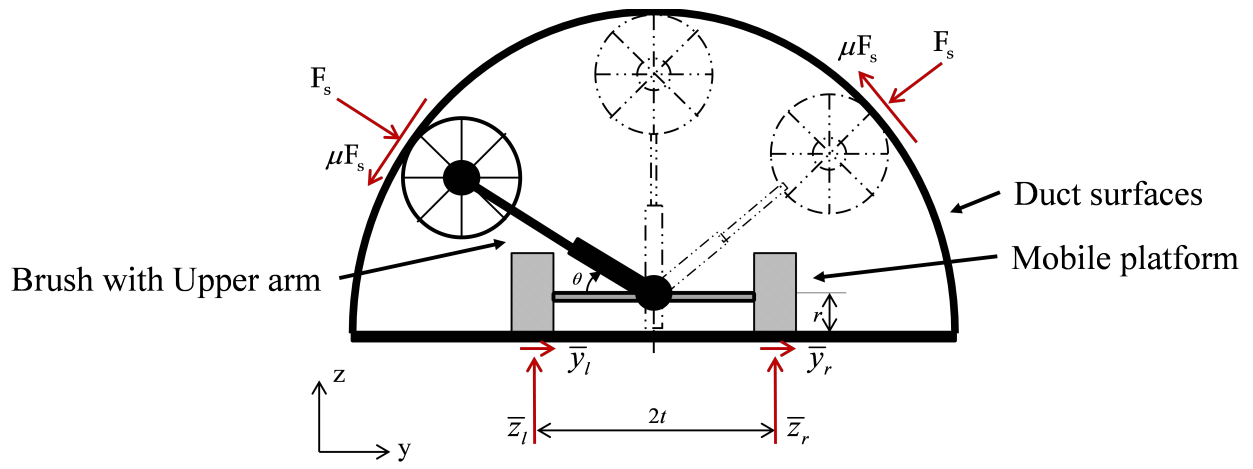

Figure 5. Free body diagram of the cleaning brush inside the circular duct.

In the free body diagram, it is assumed that the brush-interaction forces are transferred to the moving platform and wheels though the upper arm and the revolute joint. The interacting forces during the brushing in the circular duct can be expressed as the rotating angle of the upper $\operatorname{arm}(\theta)$ at time domain $(\mathrm{t})$ (see Figure 5). Consequently, the vertical $\left(\bar{F}_{z i}\right)$ and lateral $\left(\bar{F}_{y i}\right)$ forces generated by the external force are calculated with the rotation angle $\theta$ as 


$$
\begin{gathered}
\bar{F}_{z i}=\left[\begin{array}{llll}
\frac{L-a}{L} z_{l} & \frac{L-a}{L} z_{r} & \frac{a}{L} z_{r} & \frac{a}{L} z_{l}
\end{array}\right]^{T}, \\
\bar{F}_{y i}=\left[\begin{array}{llll}
\frac{L-a}{L} y_{l} & \frac{L-a}{L} y_{r} & \frac{a}{L} y_{r} & \frac{a}{L} y_{l}
\end{array}\right]^{T}
\end{gathered}
$$

where

$$
\begin{aligned}
& z_{l}=\frac{m g}{2}+\frac{F_{s} \sin \theta}{2}+\frac{\cos \theta}{2 t}\left(-F_{s} r+m_{b} g l+m_{1} g l / 2\right), \\
& z_{l}=\frac{m g}{2}+\frac{F_{s} \sin \theta}{2}+\frac{\cos \theta}{2 t}\left(F_{s} r-m_{b} g l+m_{1} g l / 2\right), \\
& f_{y}=y_{l}+y_{r}=\left\{m_{b} g l \cos \theta+t\left(z_{r}-z_{l}\right)\right\} / r
\end{aligned}
$$

where $l$ is the length of the arm, $\mu$ is the assumed friction coefficient between the brush and the duct, and $m_{b}$ and $m_{l}$ are the mass of the brush and the arm respectively. Additionally, $F_{s}$ is the normal force exerted on the surface of the duct. When the upper arm changes the rotating direction, the discontinuity of $F_{S}$ can be described by the sign function, sgn, [18] as follows

$$
\lim _{k_{s} \rightarrow \infty} \frac{2}{\pi} \tan ^{-1}\left(k_{s} \sigma\right)=\operatorname{sgn}(\sigma)
$$

Here,

$$
y= \begin{cases}1 & \text { if } \sigma>0 \\ 0 & \text { if } \sigma=0 \\ -1 & \text { if } \sigma<0\end{cases}
$$

and $k_{S}$ is a constant greater than 1 . Additionally, $F_{S}$, which is the uncertain repulsive force based on the nonlinear deformation characteristics of the brush, can be represented with a random function as follows:

$$
\begin{array}{r}
F_{s}=\left|F_{s}^{\prime}+0.05 r_{n} F_{s}^{\prime}\right|, \\
F_{s}^{\prime}=F_{p} \frac{2}{\pi} \tan ^{-1}\left(k_{s} \sin (2 \pi f t)\right)
\end{array}
$$

where $F_{p}$ is the reactive normal force to the surface, $r_{n}$ is a pseudo random function uniformly distributed between 0 and 1 , with $f$ being the reciprocating-motion frequency of the brushing arm. The equation of motion of the platform can be rewritten by including the external force obtained from the mathematical model of the brush contact from Equations (10)-(14) as

$$
\mathbf{M} \ddot{\mathbf{q}}+\mathbf{F}(\mathbf{q}, \dot{\mathbf{q}})=\mathbf{E}(\mathbf{q}) \tau+\mathbf{I}(\theta, \mathbf{q}, \dot{\mathbf{q}})
$$

where $\theta$ is the rotational angle of the upper arm. The vector of the external force $(I)$ is given by

$$
I(\theta, q, \dot{q})=\left[\begin{array}{c}
\mu_{x} \sum_{i=1}^{4} \bar{F}_{z i} \cos \phi-\sum_{i=1}^{4} \bar{F}_{y i} \sin \phi \\
+m I C R_{x} \dot{\phi}^{2} \cos \phi \\
\mu_{x} \sum_{i=1}^{4} \bar{F}_{z i} \sin \phi+\sum_{i=1}^{4} \bar{F}_{y i} \cos \phi \\
+m I C R_{x} \dot{\phi}^{2} \sin \phi \\
\bar{M}_{r}
\end{array}\right]
$$

and the additional moment exerted by the brush $\operatorname{contact}\left(\bar{M}_{r}\right)$ is

$$
\begin{aligned}
\bar{M}_{r} & =a\left(\bar{F}_{y 1}+\bar{F}_{y 2}\right)-(L-a)\left(\bar{F}_{y 3}+\bar{F}_{y 4}\right) \\
& +\left(W-W_{L}\right)\left\{R_{x 2}+R_{x 3}+\mu_{x}\left(\bar{F}_{z 2}+\bar{F}_{z 3}\right)\right\} \\
& -W_{L}\left\{R_{x 1}+R_{x 4}+\mu_{x}\left(\bar{F}_{z 1}+\bar{F}_{z 4}\right)\right\}
\end{aligned}
$$


In the calculation of $\left(\bar{M}_{r}\right)$, the center of mass (CM) location of the platform can be shifted by periodic motion of the upper arm within the length of $W_{L}$, which is the distance from the left side of the platform to the CM [9].

\section{Trajectory-Tracking Control}

The duct-cleaning process is carried out by rolling brushes on the moving platform contacting the inner duct surfaces at constant velocity. The mobile platform should be located on the reference trajectory, which is composed of points necessary for the brush to maintain pressure on the surface. Since the theory of perfect velocity tracking requires a complete dynamic model without consideration of external-disturbance effects in speed control, unknown interaction forces in the brushing mechanism can make the control system unstable by transmitting net force to wheels and the floor [19]. Therefore, a backstepping method to resolve those problems of the perfect velocity theory can be applied by a recursive process to nonlinear systems with indistinct boundedness of uncertainty [20]. Thus, the backstepping algorithm is effective in stabilizing the position error of the trajectory-tracking control in the case of existing disturbances and uncertainty of dynamic model of the platform. In the integrator backstepping method, the object velocity vector, $v_{c}$, and the derivative of the velocity can be obtained from the position errors between the reference and the platform vector, $e$, as follows [7]

$$
\begin{gathered}
\mathbf{e}=\left[\begin{array}{c}
e_{x} \\
e_{y} \\
e_{\phi}
\end{array}\right]=\left[\begin{array}{ccc}
\cos \phi & \sin \phi & 0 \\
-\sin \phi & \cos \phi & 0 \\
0 & 0 & 1
\end{array}\right]\left[\begin{array}{ll}
x_{r} & -x \\
y_{r} & -y \\
\phi_{r} & -\phi
\end{array}\right], \\
\dot{\mathbf{e}}=\left[\begin{array}{c}
-\dot{x}+\omega e_{y}+v_{r} \cos \left(e_{\phi}\right) \\
-\omega e_{x}+v_{r} \sin \left(e_{\phi}\right) \\
\dot{\phi}_{r}-\omega
\end{array}\right], \\
\mathbf{v}_{\mathbf{c}}=\left[\begin{array}{c}
v_{r} \cos \left(e_{\phi}\right)+k_{1} e_{x} \\
\dot{\phi}_{r}+k_{2} v_{r} e_{y}+k_{3} v_{r} \sin \left(e_{\phi}\right)
\end{array}\right], \\
\dot{\mathbf{v}}_{\mathbf{c}}=\left[\begin{array}{c}
v_{r} \cos \left(e_{\phi}\right) \\
\ddot{\phi}_{r}+k_{2} v_{r} e_{y}+k_{3} \dot{v}_{r} \sin \left(e_{\phi}\right)
\end{array}\right]+\left[\begin{array}{ccc}
k_{1} & 0 & -v_{r} \sin \left(e_{\phi}\right) \\
0 & k_{2} v_{r} & k_{3} v_{r} \cos \left(e_{\phi}\right)
\end{array}\right] \dot{\mathbf{e}}
\end{gathered}
$$

The control input for trajectory-tracking can be obtained by the velocity vector of the platform feedback as

$$
u=v_{c}+K_{4}\left(v_{c}-v\right)
$$

where $K_{4}$ is a positive-definite diagonal matrix $K_{4}=k_{4} I$. Error stabilization of the reference trajectory can be achieved using the velocity feedback of the platform in Lyapunov stability theory [7]. However, since the system becomes unstable when there exist unknown factors such as the friction force between wheels and the floor, nonlinear controllers (such as a neural network for inferring uncertainties) or advanced PID controllers are required to reduce the position error for the trajectory-tracking controller [9]. That is, a PD-backstepping technique can offer a good solution for improving the system stability because the previous backstepping method requires a relatively long settling time from the high iteration time to find the solution.

PD-backstepping is also adaptable to applications with hardware limitations, such as the torque capacity of the traction motor. Consequently, the control input of the PD-backstepping can be applied as the backstepping approach. Thus, the object-velocity vector $\left(\mathbf{v}_{\mathbf{c}}^{\prime}\right)$ is described as

$$
\mathbf{v}_{\mathbf{c}}^{\prime}=\left[\begin{array}{c}
v_{r} \cos \left(e_{\phi}\right)+u_{P I D, k 1} \\
\dot{\phi}_{r}+5 u_{P I D, k 2} v_{r}+\frac{1}{4} u_{P I D, k 3} v_{r} \sin \left(e_{\phi}\right)
\end{array}\right]
$$


where

$$
\begin{array}{r}
\Delta u_{P I D, k}^{\prime}=k_{p}\left[\left(e_{k}-e_{k-1}\right)+\Delta d_{k}\right], \\
\Delta d_{k}^{\prime}=(D \cdot \eta-1) d_{k-1}^{\prime}+\eta\left(e_{k}-e_{k-1}\right)
\end{array}
$$

and $e_{k}$ is the error vector of the sampling time of $k$. The effective energy is generated and the harmonic wave in the control input is eliminated by applying a low pass filter to the discrete form of the derivative controller, $\Delta d_{k}$, generating effective energy. Additionally, $u_{P I D}^{\prime}$ is of incremental form to prevent rapid changes of the tractive torque required in the controller. The control scheme of the PD-backstepping controller is illustrated in Figure 6. The red section in Figure 6 is the integration of PD control and backstepping method where the system variable is treated as an independent input for subsystem and each step results are updated for the control of the next step.

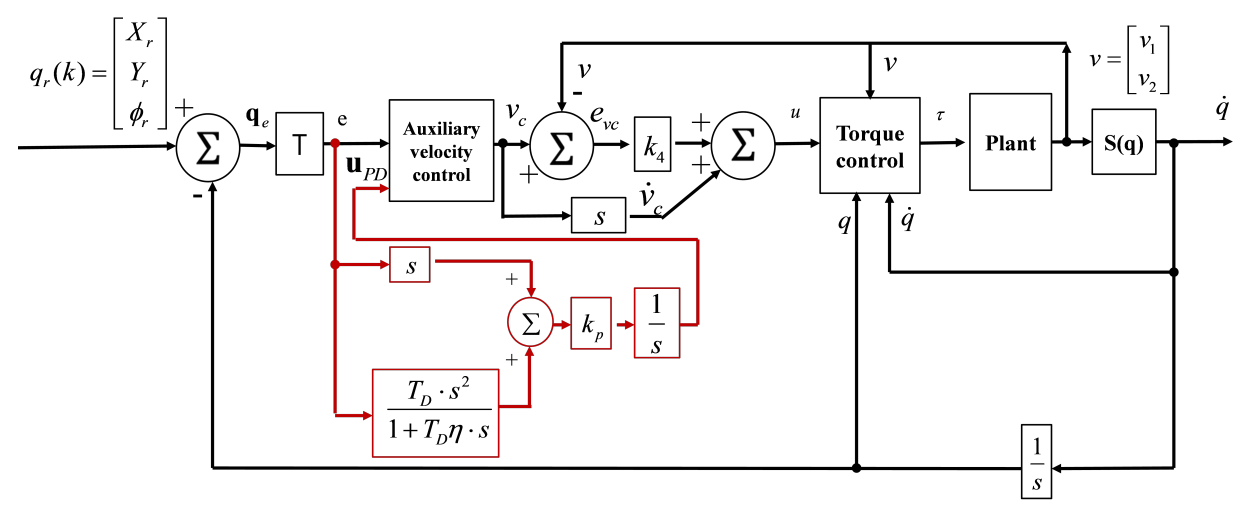

Figure 6. A control scheme of PD-backstepping for the trajectory tracking controller.

\section{Results}

\subsection{Numerical Analysis}

The performance of the PD-backstepping-based trajectory-tracking controller has been evaluated by numerical method using a MATLAB ${ }^{T M}$ tool. Actual contacting mechanism between brush and inner surface of the duct is complicated because the brush consists of many flexible filaments that bend during cleaning. Therefore, a model of the complicated brush-contacting mechanism on the surface of the duct has been estimated and simplified by assuming point contact between brush and duct surface as modeled in Figure 5. Modeling parameters of the platform are summarized in Table 1.

Table 1. Summary of modeling parameters in the numerical simulation.

\begin{tabular}{ccc}
\hline Description & Symbol & Values \\
\hline Platform Size & $L$ & $0.3 \mathrm{~m}$ \\
CM to front wheel & $a$ & $0.15 \mathrm{~m}$ \\
Distance b/w left and right wheel & $w$ & $0.23 \mathrm{~m}$ \\
Wheel radius & $r$ & $0.05 \mathrm{~m}$ \\
Math moment of Inertia & $I$ & $0.19 \mathrm{~kg} \cdot \mathrm{m}^{2}$ \\
Overall mass & $m$ & $7.823 \mathrm{~kg}$ \\
Longitudinal Friction coefficient & $\mu_{x}$ & 0.5 \\
Lateral friction coefficient & $\mu_{y}$ & 0.1 \\
Initial position of the platform & $X_{0}, Y_{0}$ & $-0.02 \mathrm{~m}, 0.05 \mathrm{~m}$ \\
Max. torque of the traction motor & $T_{m a x}$ & $0.95 \mathrm{Nm}$ \\
\hline
\end{tabular}

The reference trajectory was set to $\left[X_{r}, Y_{r}\right]=\left[v_{r} t, 0.2 \sin (2 \pi f t / 60)\right]$. The friction coefficients between wheels and the floor of the duct are changeable depending upon the surface status and environmental factors. However, the performance of the controller was evaluated by estimating the driving torque based on the arbitrary friction coefficients listed in Table 1. 
Tractive torques were calculated to reduce the position error of the platform by considering the frictional behavior of the wheels. A maximum torque limit was applied to the traction motors of the platform. Figure 7 shows the analytical results of the motor torque distribution at each wheel when various interaction forces ranging from $20 \mathrm{~N}$ to $40 \mathrm{~N}$ were applied to the rotating brushes. Figure 8 shows the position errors at the reference trajectory. The overall position errors increase when lateral and vertical forces due to the contacting-brush pressure act on each wheel. In particular, lateral-position errors are affected by the lateral force due to the brush-contacting mechanism. The yaw moment based on the torque differential between the left and right wheels can be used to adjust the heading angle to the reference trajectory with the nonholonomic constraint. The driving force is calculated in proportion to the vertical force exerted on the wheel. The torque difference between the left and right wheels increases as the pressurizing force used in brushing increases.
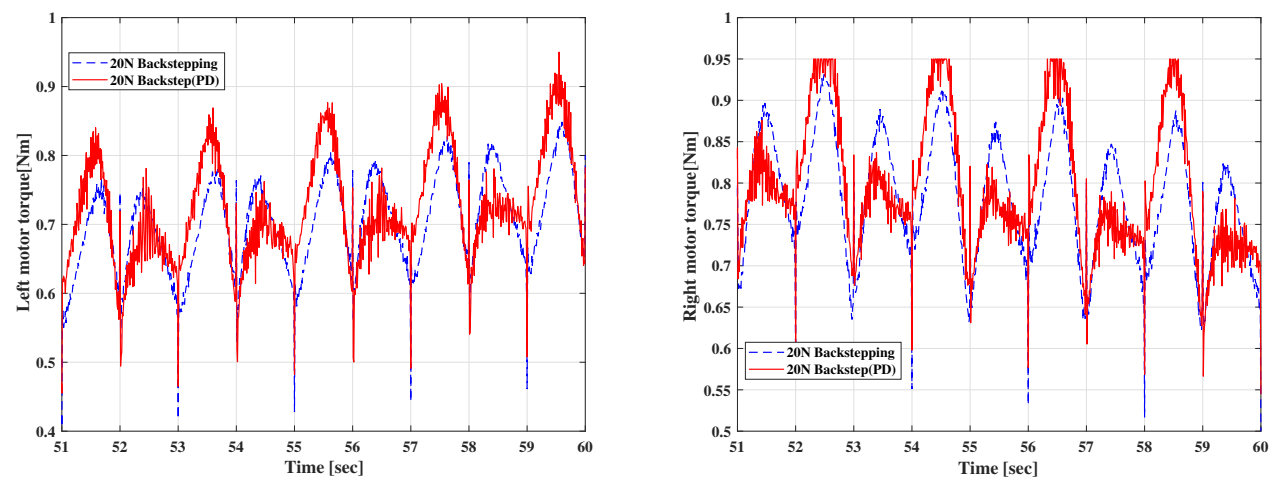

(a)
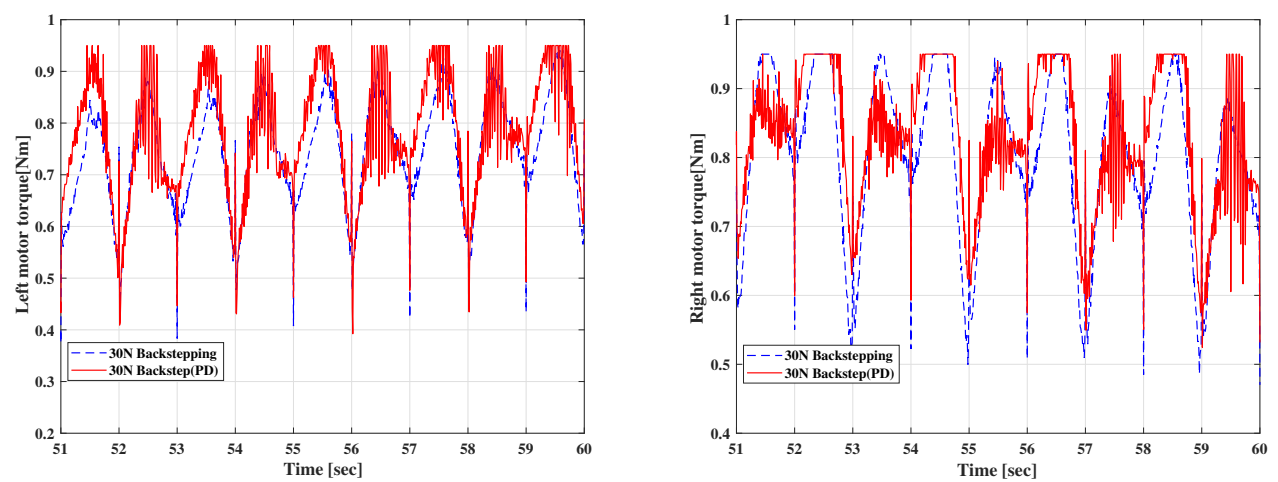

(b)
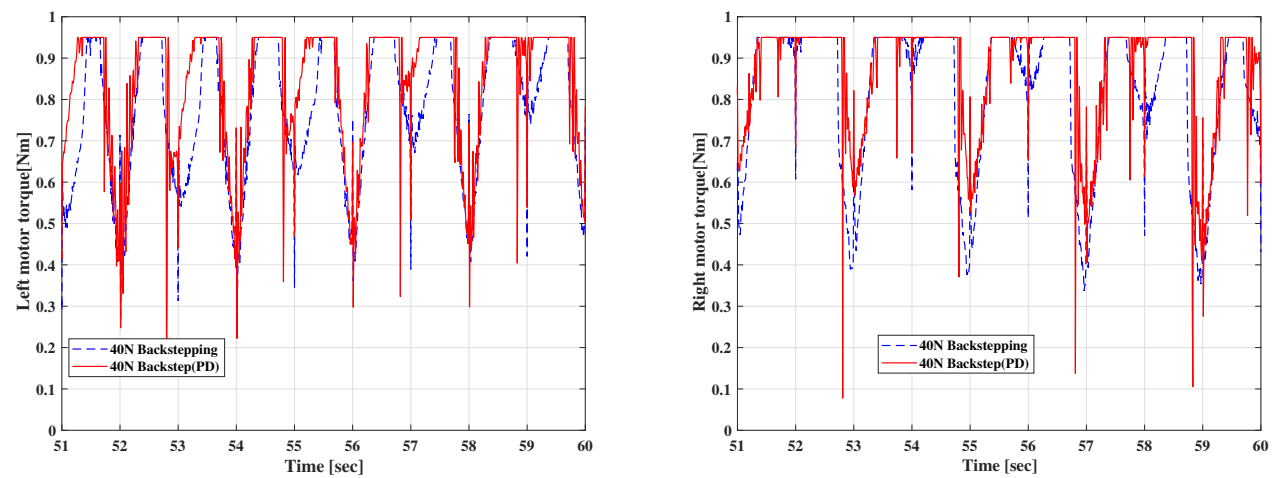

(c)

Figure 7. Results of the motor torque with variable brush interaction force. (a) Force $=20 \mathrm{~N}$; (b) Force $=30 \mathrm{~N}$; (c) Force $=40 \mathrm{~N}$. 
Figure 7 indicates in the $\mathrm{x}$-directional errors, which can be settled by applying both backstepping and PD-backstepping controllers. However, when larger pressuring forces over $30 \mathrm{~N}$ act on the brush, the control-torque inputs reach the upper limit of the allowed torque value, $0.95 \mathrm{Nm}$ as shown in Figure $7 \mathrm{~b}, \mathrm{c}$. Thus, there exist overshoots of about $20 \mathrm{~mm}$ when the backstepping controller is applied as depicted in Figure 8b, and the errors become unstable as the brush pressure increases to $40 \mathrm{~N}$, as shown in Figure 8c. When the PD-backstepping controller is applied, the trajectory-tracking control works in the case of a large brush pressure of $40 \mathrm{~N}$ and the settling time decreases from 25 to $8 \mathrm{~s}$, as shown in Figure 8.
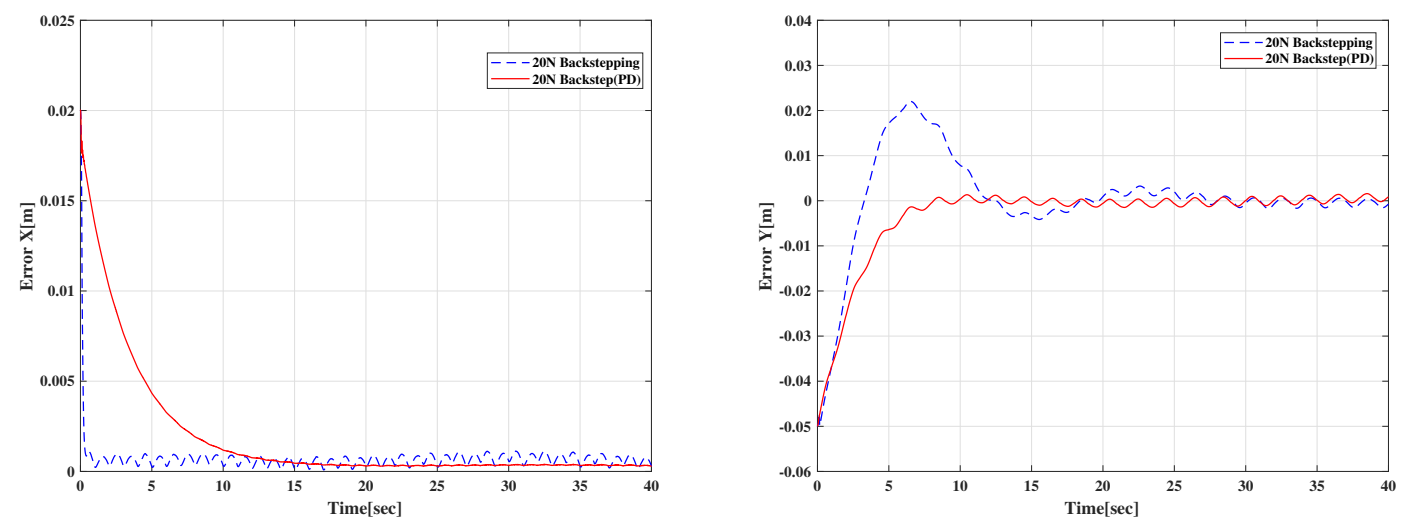

(a)
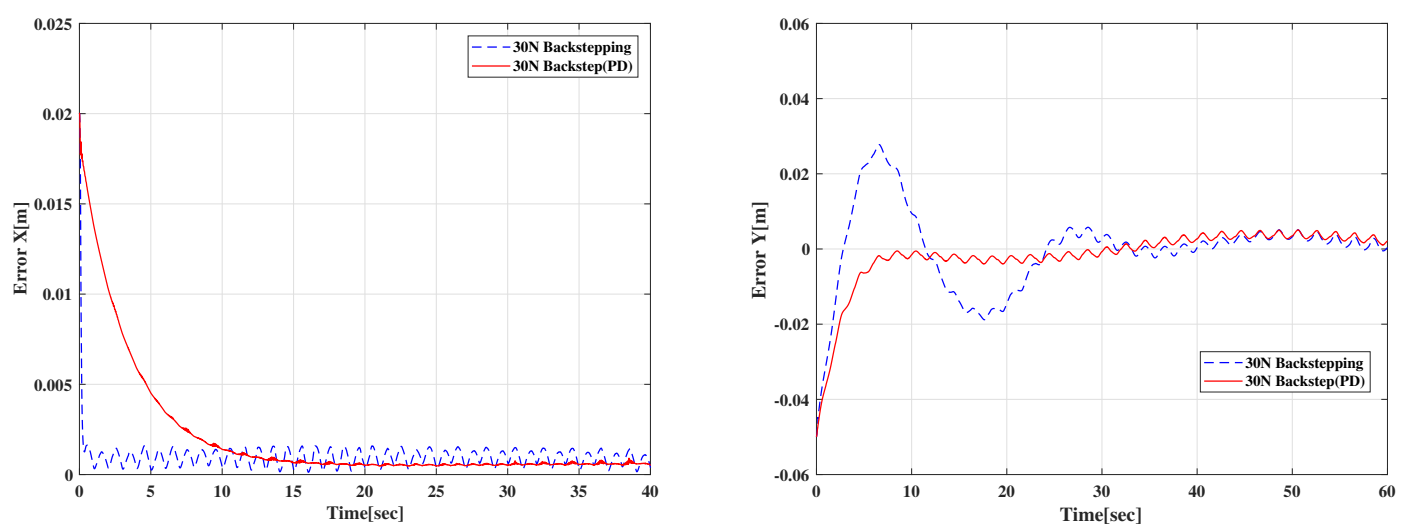

(b)
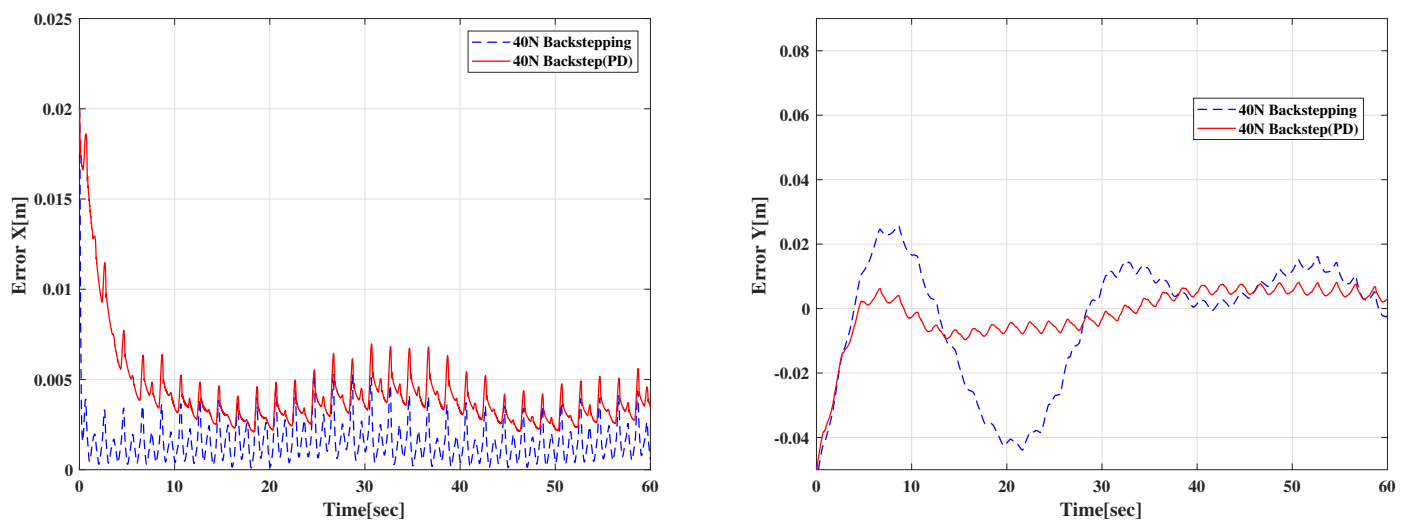

(c)

Figure 8. Position errors with variable brush interaction forces. (a) Force $=20 \mathrm{~N}$; (b) Force $=30 \mathrm{~N}$; (c) Force $=40 \mathrm{~N}$. 
In addition, compared to the backstepping-control method, the PD-backstepping controller can reduce the velocity errors by $50 \%$ at a speed of $0.05 \mathrm{~m} / \mathrm{s}$ as shown in Figure 9 . Figure 10 represents the analytical results of the longitudinal position of the ICR and the wheel slippage. By applying a limited torque of $0.95 \mathrm{Nm}$ to the wheel, more traction force is required to stabilize the movement of the platform corresponding to the lateral force and the yawing moment exerted by the brush-interaction force. Therefore, the higher pressure force on the brush induces wheel slippage and shifts the location of the ICR in the longitudinal direction. Despite of the limitations of motor torque, stable tracking control can be accomplished by applying the PD-backstepping control technique, especially under a high brushing force of $40 \mathrm{~N}$.

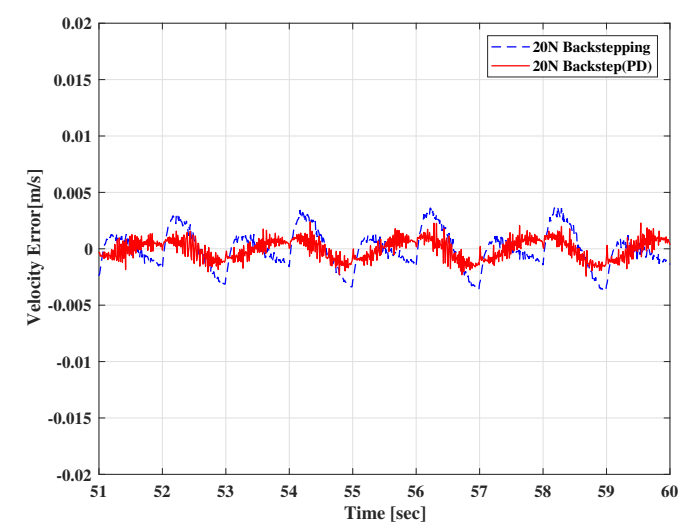

(a)

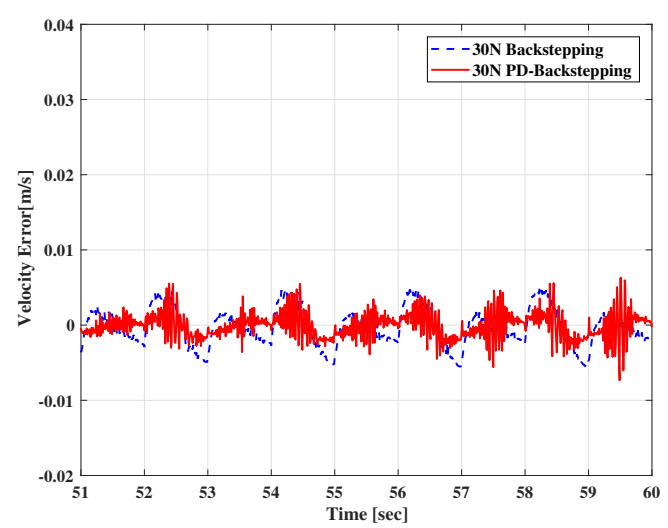

(b)

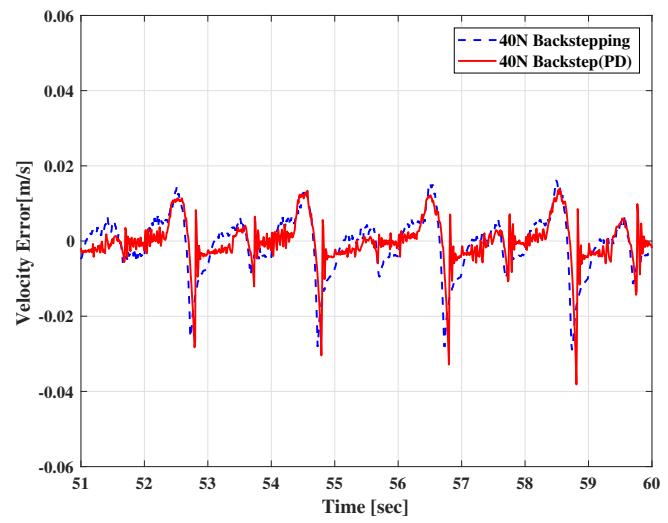

(c)

Figure 9. Velocity errors with variable brush interaction forces. (a) Force $=20 \mathrm{~N}$; (b) Force $=30 \mathrm{~N}$; (c) Force $=40 \mathrm{~N}$. 

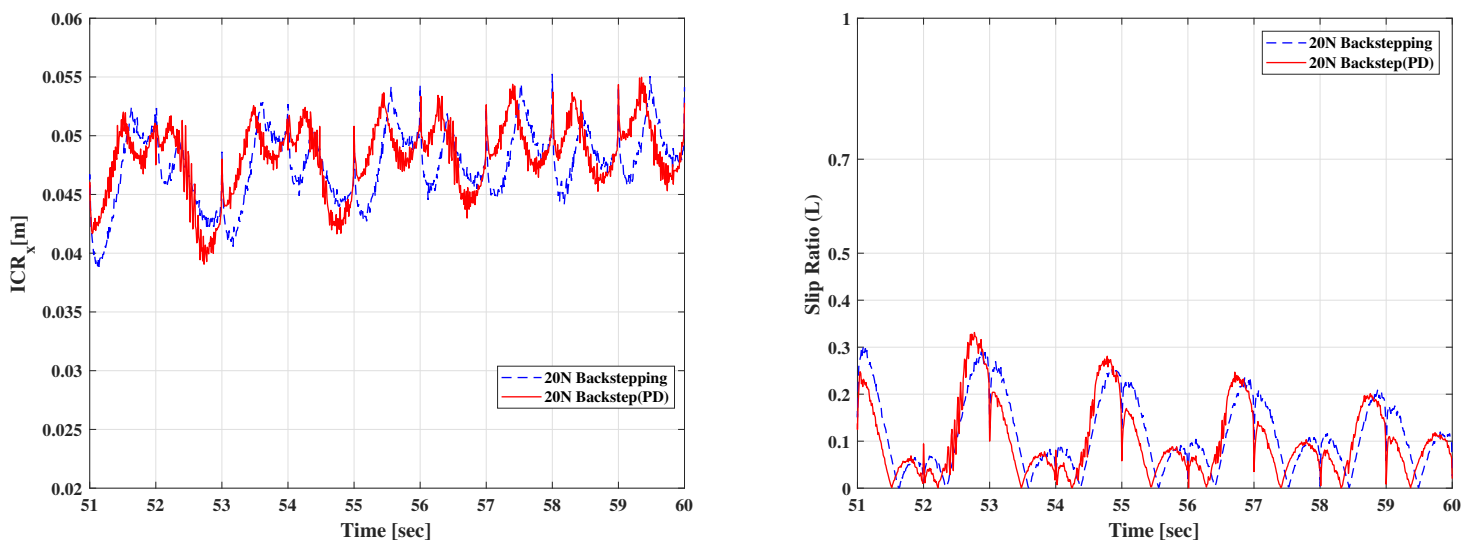

(a)
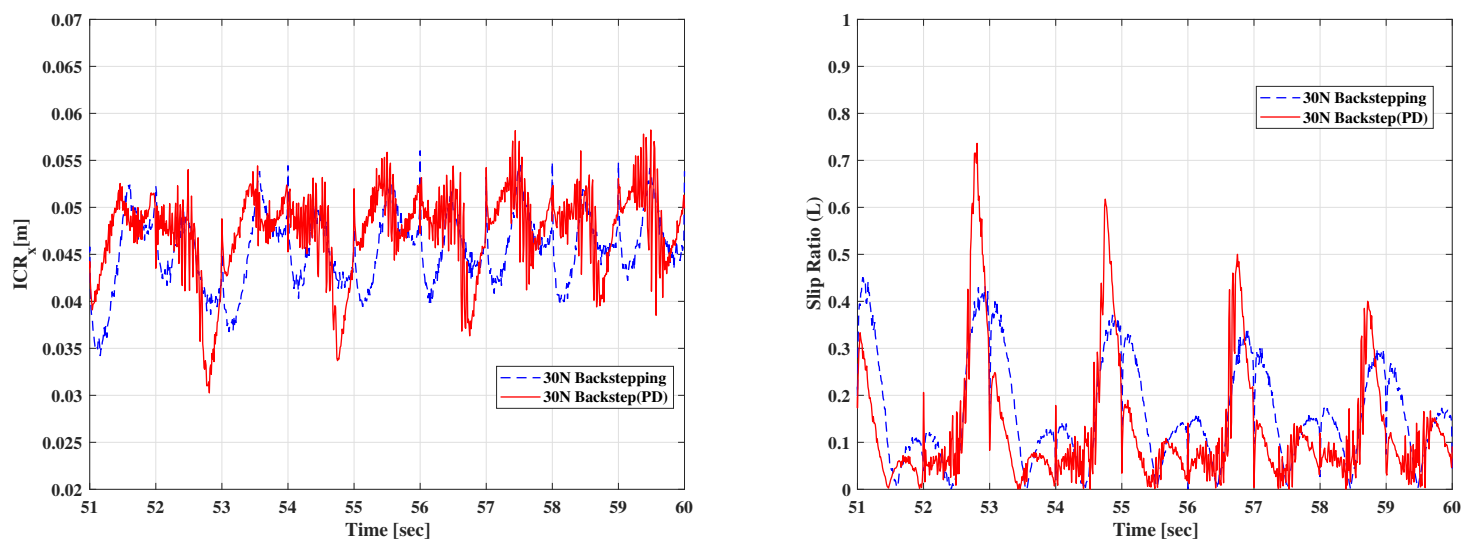

(b)
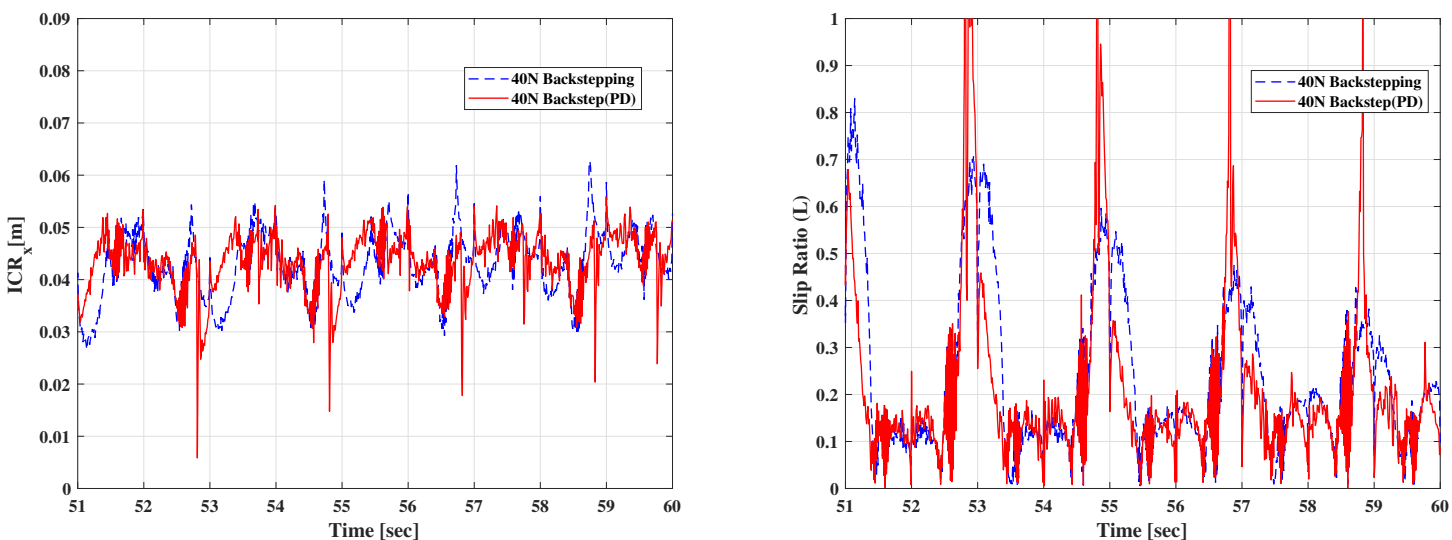

(c)

Figure 10. Position of the $I C R_{x}$ and front-left wheel slippage with variable brush interaction force. (a) Force $=20 \mathrm{~N}$; (b) Force $=30 \mathrm{~N}$; (c) Force $=40 \mathrm{~N}$.

Figure 11 shows the position errors under $20 \mathrm{~N}$ of brushing pressure as the period of reciprocating motion of the upper arm changes from 2 to $1 \mathrm{~s}$. The overshoot and fluctuation of the position error due to the periodic slippage of the platform in the lateral direction can be reduced as the period of upper-arm motion decreases. While 10 20 $\mathrm{mm}$ of overshoot and about $25 \mathrm{~s}$ of settling time are required in the backstepping control scheme, the PD-backstepping controller retenuates the overshoot and stabilizes the position errors for up to $8 \mathrm{~s}$. However, the velocity errors increase as the period of reciprocating motion in the PD-backstepping controller becomes short, as shown in Figure 12. 

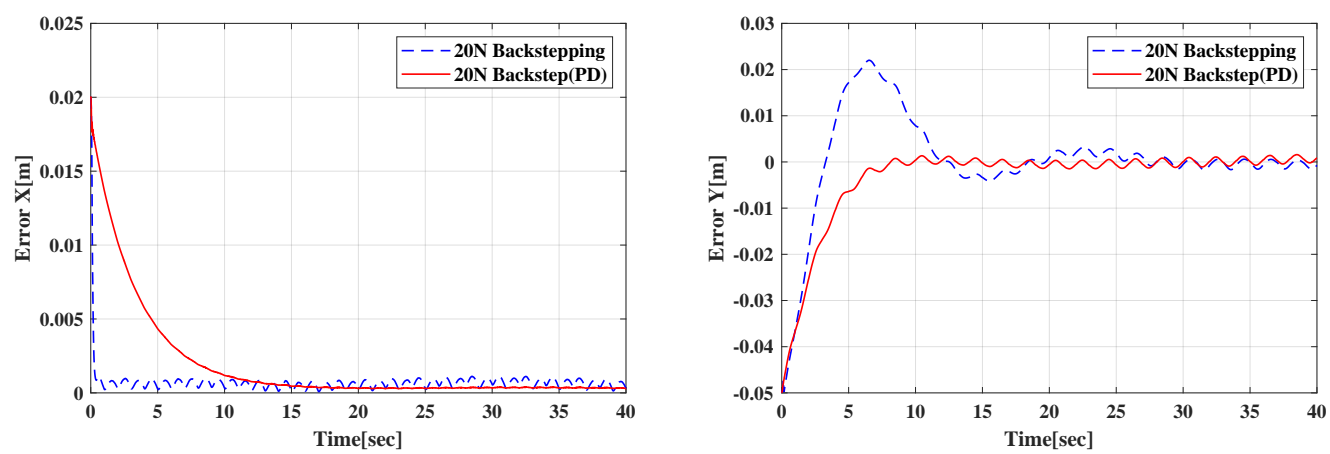

(a)
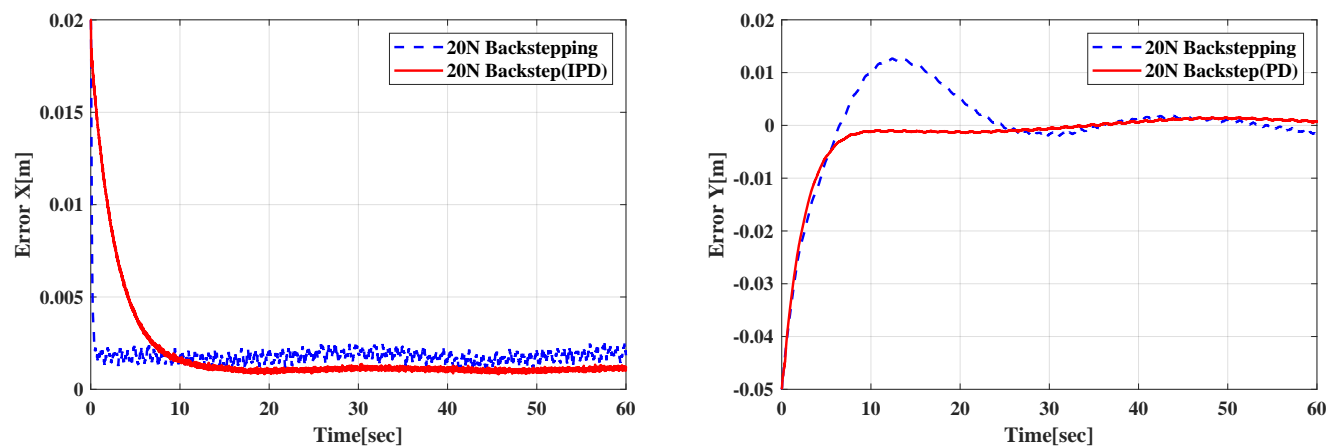

(b)
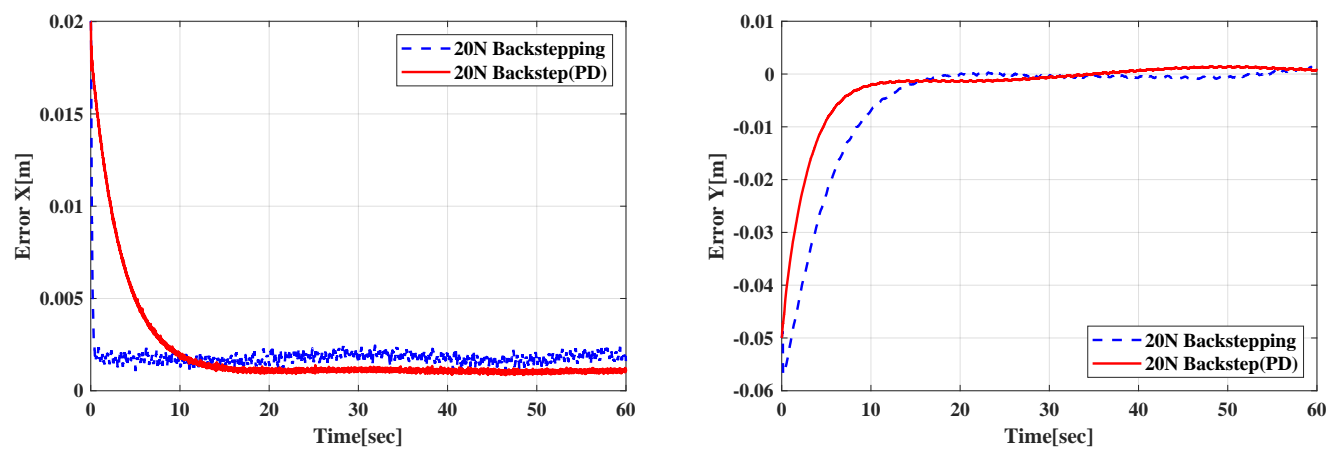

(c)

Figure 11. Position errors with reciprocal motion of the upper arm. (a) Period $=2.0 \mathrm{~s}$; (b) Period $=1.5 \mathrm{~s}$; (c) Period $=1.0 \mathrm{~s}$.

\subsection{Experimental Results}

The performance of the trajectory-tracking controller was examined in an air-ventilation duct by applying random external forces to the platform. Random periodic force was used in the uncertain repulsive-brushing-pressure mechanism applied by the upper-arm motion and the system model through the duct surface and wheels. In addition, the velocity of the mobile platform was controlled with the backstepping and PD-backstepping controllers during the brushing process.

Figure 13 shows the results of the trajectory tracking control experiment when external force was applied to each wheel. Current values at traction represent the traction loads acting on each wheel. The external force on the wheels causes tracking errors between the platform and the reference trajectory. Therefore, the controller increases the motor-torque inputs to assign the yaw motion to compensate for heading-angle errors of the platform. Since the PD-backstepping controller increases torque inputs proportionally to the motor constant, heading-angle errors have converged rapidly to zero degrees. In addition, the velocity was maintained at $50 \mathrm{~mm} / \mathrm{s}$ as the desired speed of the moving platform (Figure 13b). The experimental results also shows that the PD-backstepping method improves 
system stability by minimizing trajectory tracking errors during the changes of brushing force, and the motor torques are more adaptable to the external force in case of PD-backstepping method.

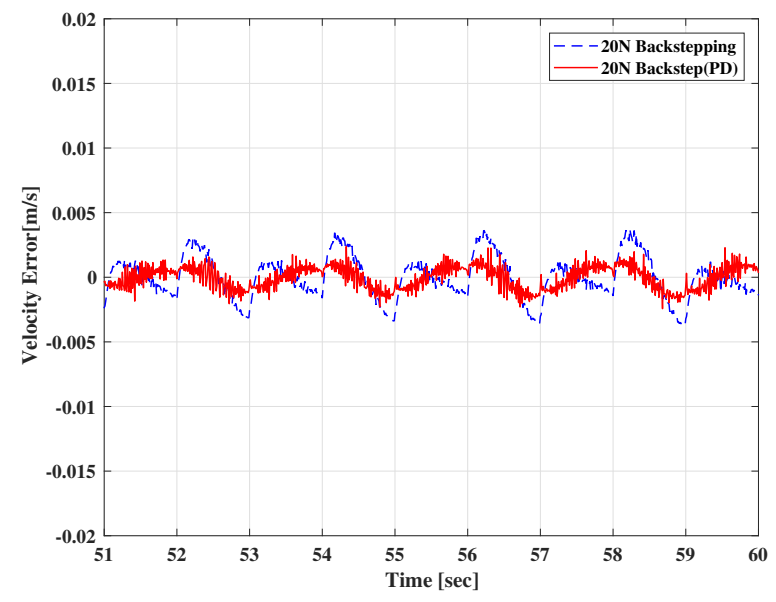

(a)

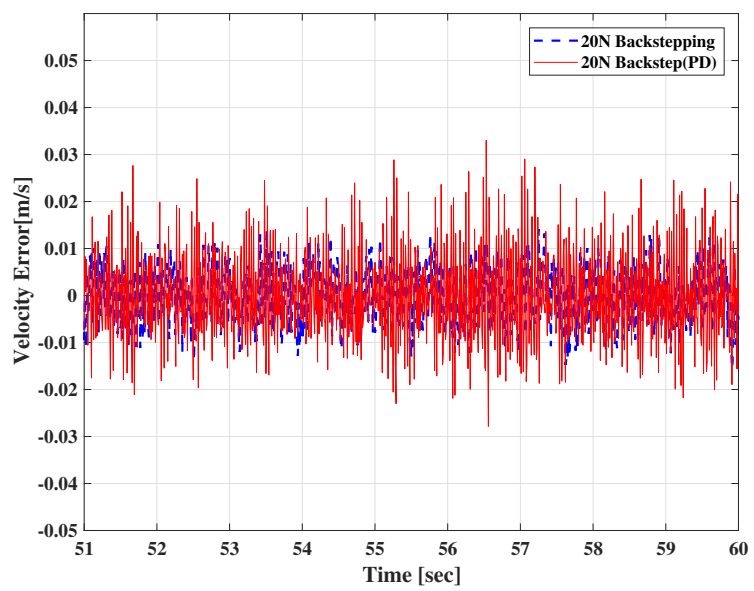

(b)

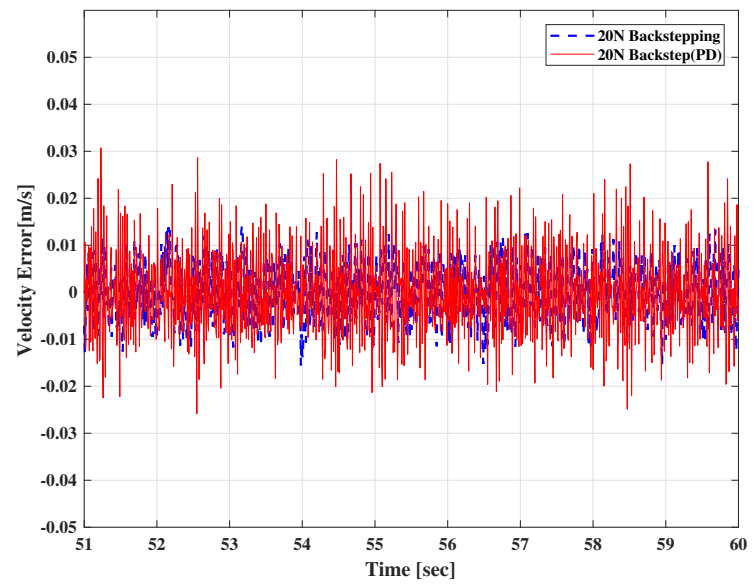

(c)

Figure 12. Velocity errors with reciprocal motion of the upper arm. (a) Period $=2.0 \mathrm{~s} ;(\mathbf{b})$ Period $=1.5 \mathrm{~s}$; (c) Period $=1.0 \mathrm{~s}$. 


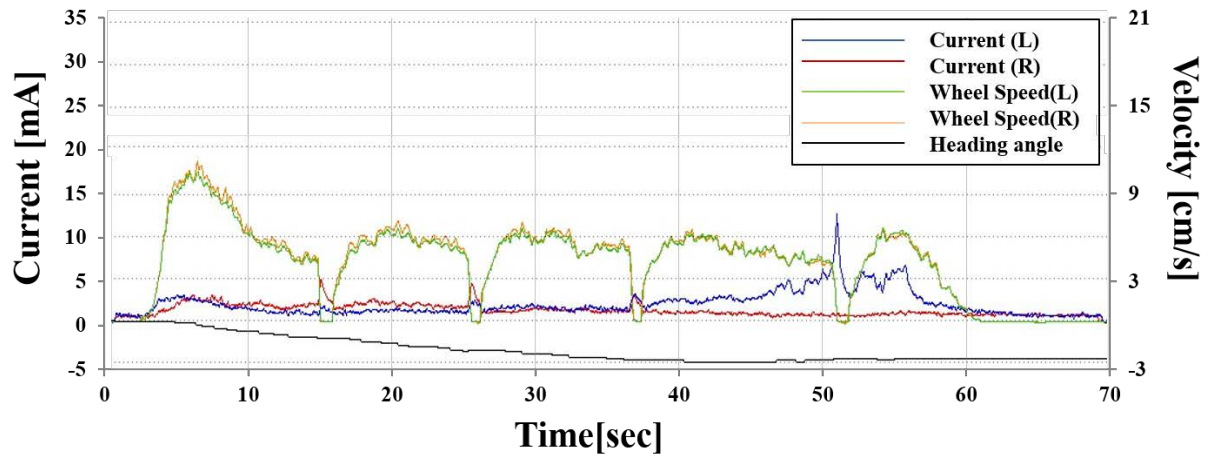

(a)

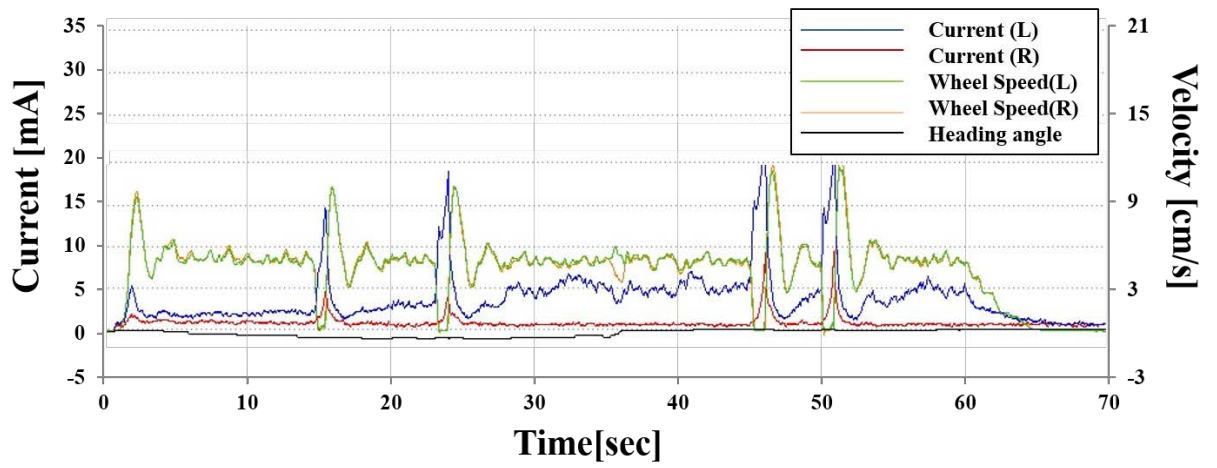

(b)

Figure 13. Experimental results of trajectory tracking control. (a) Backstepping control; (b) PD-backstepping control.

\section{Conclusions}

In air-duct-cleaning applications under uncertain external cleaning forces, trajectory-tracking control based on modeling of the force acting on a mobile platform has been conducted using the PD-backstepping algorithm. The proposed controller is based on an incomplete-dynamic model of the platform without processing of nonlinear inference. In addition, a four-wheeled skid-steering platform model has been constructed by considering wheel slippages and the location shift of the ICR. The motor torque required by the controller can be calculated to adjust the path considering the unmeasurable force generated by the brush contact acting on the wheel without singularity of the arm movement. As the pressurizing force exerting on the brush approaches $40 \mathrm{~N}$, the maximum torque limit of the motor, the skid-steered platform model based the PD-backstepping controller has enabled stable tracking control. Furthermore, when the reciprocating-motion velocity of the upper arm is increased to reduce the cleaning time, the settling time can be reduced by implementing the PD-backstepping controller.

The experimental results show that trajectory tracking was achieved by adopting the suggested controller to generate suitable motor torque to reduce the position error of the platform while maintaining the objective speed. However, the inertial force from the mass of the upper-arm mechanism can be additionally produced at the directional changing points of the cleaning arm. Therefore, the inertial-force model of rapid movement of the arms can be considered to provide robust control.

Author Contributions: Data curation, S.J.; Formal analysis, D.J.; Investigation, W.J.; Methodology, W.J.; Resources, S.J.; Supervision, W.J.

Funding: This research was supported by a grant from R\&D program of the Korea Railroad Research Institute, Republic of Korea.

Conflicts of Interest: The authors declare no conflict of interest. 


\section{References}

1. Jeong, W. Performance Analysis of a Mobile Duct-cleaning Robot. Int. J. Adv. Appl. 2014, 7, 26-32

2. Chwa, D. Sliding-Mode Tracking Control of Nonholonomic Wheeled Mobile Robots in Polar Coordinates. IEEE Trans. Control Syst. Technol. 2004, 12, 637-644 [CrossRef]

3. Caracciolo, L. Trajectory-tracking Control of a Four-Wheel Differentially Driven Mobile Robot. In Proceedings of the IEEE International Conference on Robotics and Automation (ICRA), Detroit, MI, USA, 10-15 May 1999; pp. 2632-2638.

4. Yi, J.; Song, D.; Zhang, J.; Goodwin, Z. Adaptive trajectory-tracking control of skid-steered mobile robots. In Proceedings of the IEEE International Conference on Robotics and Automation(ICRA), Rome, Italy, 10-14 April 2007; pp. 2605-2610.

5. Piltan, F.; Sulaiman, N.; Soltani, S.; Marhaban, M.H.; Ramli, R. An Adaptive sliding surface slope adjustment in PD Sliding Mode Fuzzy Control for Robot Manipulator. Int. J. Control Autom. 2011, 4, 65-76.

6. De Wit, C.C.; Khennouf, H.; Samson, C.; Sordalen, O.J. Nonlinear control design for mobile robots. In Recent Trends in Mobile Robots; Zheng, Y.F., Ed.; World Scientific: Singapore, 1993; pp. 121-156.

7. Fierro, R.; Lewis, F.L. Control of a Nonholonomic Mobile Robot: Backstepping Kinematics into Dynamics. J. Robot. Syst. 1997, 14, 149-163. [CrossRef]

8. Shin, J. Robust Adaptive Fuzzy Backstepping Control for Trajectory-tracking of an Electrically Driven Nonholonomic Mobile Robot with Uncertainties. J. Inst. Control Robot. Syst. 2012, 18, 902-911. [CrossRef]

9. Jeon, S.; Jeong, W. The Stable Trajectory-tracking Control of a Skid-steered Mobile Platform with Dynamic Uncertainties. Int. J. Adv. Robot. Syst. 2015, 12, 1-9. [CrossRef]

10. Yu, J.; Chang, J. A new adaptive backstepping control algorithm for motion control systems. In Proceedings of the 2000 IEEE International Conference on Control Applications, Anchorage, AK, USA, 25-27 September 2000; pp. 367-372.

11. Benaskeur, A.; Desbiens, A. Backstepping-based adaptive PID control. IEE Proc. Control Theory Appl. 2002, 149, 54-59. [CrossRef]

12. Kwan, C.M.; Lewis, F.L. Robust backstepping control of induction motors using neural networks. IEEE Trans. Neural Netw. 2000, 11, 1178-1187. [CrossRef] [PubMed]

13. Yu, W.; Collins, E.G. Analysis and Experimental Verification for Dynamic Modeling of A Skid-steered Wheeled Vehicle. IEEE Trans. Robot. 2010, 26, 340-353. [CrossRef]

14. Martinez, J.L.; Mandow, A.; Morales, A.; Pedraza, S.; Garcia-Cerezo, A. Approximating Kinematics for Tracked Mobile Robots. Int. J. Robot. Res. 2005, 24, 867-878. [CrossRef]

15. Yi, J.; Wang, H.; Zhang, J.; Song, D.; Jayasuria, S.; Liu, J. Kinematic Modeling and Analysis of Skid-Steered Mobile Robots With Applications to Low-Cost Inertial-Measurement-Unit-Based Motion Estimation. IEEE Trans. Robot. 2009, 25, 1087-1097.

16. Wu, X.; Xu, M.; Wang, L. Differential Speed Steering Control for Four-Wheel Independent Driving Electric Vehicle. Int. J. Mater. Mech. Manuf. 2013, 1, 355-359.

17. Le, A.T. Modeling and Control of Tracked Vehicles. Ph.D. Dissertation, University of Sydney, Sydney, Australia, 1999.

18. Kozlowski, K.; Pazderski, D. Modeling and control of a 4-wheel skid-steering mobile robot. Int. J. Appl. Math. Comput. Sci. 2004, 14, 477-496.

19. Kanayama, Y.; Kimura, Y.; Miyazaki, F.; Noguchi, T. A Stable Tracking Control Method for an Autonomous Mobile Robot. In Proceedings of the IEEE International Conference on Robotics and Automation (ICRA), Cincinnati, OH, USA, 13-18 May 1990; pp. 384-389.

20. Khalil, H.K. Nonlinear Systems, 2nd ed.; Prentice Hall: Upper Saddle River, NJ, USA, 1996.

(C) 2019 by the authors. Licensee MDPI, Basel, Switzerland. This article is an open access article distributed under the terms and conditions of the Creative Commons Attribution (CC BY) license (http:/ / creativecommons.org/licenses/by/4.0/). 